\title{
First-Principles Study on Electronic, Magnetic and Elastic Properties of $\mathrm{CeN}$ and PmN.
}

\author{
Gomaa.M. Elalfy ${ }^{a}$, Reham. M.Shabara ${ }^{a}$, Samy H. Aly ${ }^{a}$, \\ and Sherif Yehia ${ }^{b}$ \\ ${ }^{a}$ Department of Physics, Faculty of Science at Damietta, Damietta \\ University, P.O. Box 89, New Damietta 34517, Egypt. \\ ${ }^{b}$ Faculty of Science and Arts at Al-Ola Physics Department \\ P. O. Box: 344 Taibah University, Saudi Arabia. \\ E-mail: Gomaa M. Elalfy (alalfygouma@yahoo.com).
}

We have used the Full Potential Nonorthogonal Local-Orbital minimum basis method (FPLO) in the Local Spin Density Approximation (LSDA) to calculate the electronic, magnetic and elastic properties of CeN and PmN. We predicted a phase transition from Rock Salt structure (RS) to Cesium Chloride (CsCl) structure in both CeN and PmN compounds at pressures of 40.7 and 42 GPa respectively. The half-metallic property was found only in the RS phase of PmN. The CeN compound, however, shows a metallic behavior in the two structures. The calculated bulk modulus of CeN and PmN in RS structure are 170.02 GPa and 154.6 GPa respectively. For PmN the energy gap and the total magnetic moment are $0.56 \mathrm{eV}$ and $4 \mu_{B}$ respectively.

\section{Introduction}

The rare-earth nitrides RENs offer interesting magnetic properties in the field of spintronics applications [1-5]. The unfilled and highly localized f-orbital atoms are responsible for the interesting structural and magnetic properties of these compounds. All RENs exhibit strong magnetic ordering because of the interaction between the partially filled rare earth $4 \mathrm{f}$ orbital, the $5 p$ valance on the $\mathrm{N}$ and $5 \mathrm{~d}$ conduction band. All RENs undergo structural transformations when pressure is applied, and many compounds transform to the $\mathrm{CsCl}$ structure under high pressure. The reason is that the reduction of the lattice dimensions, upon applying pressure, causes the interionic coulomb interaction to favor the $\mathrm{CsCl}$ structure over the rock salt structure. In general, compounds with high ionicity values are expected to have low transition pressures. The magnetic structure of the rare earth nitrides is not always known. Szotek et al [6] have performed first- principles calculations using SIC-LSD method for all rare earth nitrides in the ferromagnetic ordering. They predicted that most of light rare earth nitrides are half-metallic, and only $\mathrm{CeN}$ is a metallic compound. TbN, DyN and HoN compounds have an insulating character with a narrow gap. GdN is the most studied compound of the rare earth nitrides, where it has a half-filled Gd $4 \mathrm{f}$ shell and the highest Curie temperature among these compounds. 
Almost all of the rare earth nitrides undergo phase transitions under different pressures. Both of $\mathrm{SmN}$ and $\mathrm{EuN}$ undergoe transition from $\mathrm{NaCl}$ structure to $\mathrm{CsCl}$ structure at high pressure. Pagare et al [7] calculated a phase transition pressure of $8.5 \mathrm{GPa}$ and $14.6 \mathrm{GPa}$ for $\mathrm{SmN}$ and $\mathrm{EuN}$ (with $17.5 \%$ and $14.9 \%$ volume collapse respectively) using the Self-Consistent Tight-Binding Linear Muffin Tin Orbital method (TBLMTO) based on the density functional theory [8,9]. The $\mathrm{RS}$ to $\mathrm{CsCl}$ phase transitions in $\mathrm{YbN}$ and $\mathrm{LaN}$ were reported to take place at pressures of $133 \mathrm{GPa}$ and $100 \mathrm{GPa}$ with volume change of $5.1 \%$ and $2.6 \%$ respectively according to Rukmangad et al [10] by using two-body interionic potential theory. The transition pressure of $\mathrm{CeN}$ was found to be 62 $\mathrm{GPa}$ by Svane et al [11]. Also $\mathrm{PmN}$ transform into $\mathrm{CsCl}$ structure from $\mathrm{NaCl}$ structure at $3.4 \mathrm{GPa}$ with a volume collapse of $20 \%$ [12]. Srivastava et al [13] using the Tight-Binding Linear Muffin Tin Orbital (TBLMTO) method within the local density approximation predicted a phase transition in $\mathrm{GdN}$ from $\mathrm{NaCl}$ structure to $\mathrm{CsCl}$ structure at $30.4 \mathrm{GPa}$ with $\mathrm{V} / \mathrm{V}_{\mathrm{o}}=0.845$ for $\mathrm{NaCl}$ and 0.696 for $\mathrm{CsCl}$. Another theoretical study by Shabara et al [14], based on DFT using the Local Spin Density Approximation (LSDA) $[15,16]$ and the Generalized Gradient Approximation (GGA) [17,18] approximations, have found the phase transition pressure in $\mathrm{GdN}$ from $\mathrm{RS}$ into $\mathrm{CsCl}$ structure to be $93 \mathrm{GPa}$ and $113 \mathrm{GPa}$ respectively. The phase transition from $\mathrm{RS}$ to $\mathrm{CsCl}$ in $\mathrm{SmN}$ was predicted to take place at $8.6 \mathrm{GPa}$ by Pagare et al [7] using the first tight binding linear muffin tin orbital method. Huleil et al [19] using the Full Potential Nonorthogonal LocalOrbital minimum basis method (FPLO), within the LSDA and GGA approximations, predicted a phase transition in $\mathrm{SmN}$ from $\mathrm{RS}$ to $\mathrm{CsCl}$ at pressure of $49 \mathrm{GPa}$.

In this study, we present first-principles calculations of the electronic, magnetic and elastic properties of $\mathrm{PmN}$ and $\mathrm{CeN}$ using the electronic structure code FPLO.09. In addition, we predicted pressure induced phase transition in both compounds. The density of states and energy band are calculated for PmN and CeN compounds.

\section{Theory and Computation}

Our study is a first- principles based calculation of the elastic and magnetic properties of $\mathrm{PmN}$ and $\mathrm{CeN}$ compounds in the two crystallographic structures; $\mathrm{CsCl}$ and $\mathrm{RS}$, within the framework of density functional theory (DFT). The theoretical values of equilibrium lattice constant, magnetic moment, energy gap ,density of states (DOS) and band structures are calculated from the electronic structure by the full-potential local-orbital minimum-basis code (FPLO), to solve the Kohn-Sham equations on a regular lattice using the local spin density approximation (LSDA) .The bulk modulus and its pressure derivative have been computed using the modified Birch-Murnghan equation of state [20-22]. We have used equation (1) for fitting volume vs. energy data obtained from our ab-initio calculation: 


$$
E(V)=E_{0}+\frac{9 B_{0} V_{0}}{16}\left[B_{1}\left\{\left(\frac{V_{0}}{V}\right)^{\frac{2}{3}}-1\right\}^{3}+\left\{\left(\frac{V_{0}}{V}\right)^{\frac{2}{3}}-1\right\}^{2}\left\{6-4\left(\frac{V_{0}}{V}\right)^{\frac{2}{3}}\right\}\right]
$$

and the pressure is given by

$$
P(V)=\frac{3 B_{0}}{2}\left[\left(\frac{V_{0}}{V}\right)^{\frac{7}{3}}-\left(\frac{V_{0}}{V}\right)^{\frac{5}{3}}\right]\left[1+\frac{3}{4}\left(B_{1}-4\right)\left(\left(\frac{V_{0}}{V}\right)^{\frac{2}{3}}-1\right)\right]
$$

where $\mathrm{V}_{0}$ is the equilibrium volume, $\mathrm{E}_{0}$ the equilibrium energy, $\mathrm{B}_{0}$ the bulk modulus, and $\mathrm{B}_{1}$ the first derivative of $\mathrm{B}_{0}$ with respect to pressure.

The parameters used in FPLO run are: the k-mesh subdivision: $24 \times 24 \times 24$, the accuracies of the density and total energy are $10^{-6} \AA^{-3}$ and $10^{-8}$ Hartree respectively. For the RS structure, the space group is (\#225) and the atomic positions are $(0,0,0)$ for $\mathrm{Pm}(\mathrm{Ce})$ and $(1 / 2,1 / 2,1 / 2)$ for $\mathrm{N}$. The atoms in the $\mathrm{CsCl}$ structure are located at $(0,0,0)$ for $\mathrm{Pm}(\mathrm{Ce})$ and $(1 / 2,1 / 2,1 / 2)$ for $\mathrm{N}$ atom with space group (\#221).

\section{Results and Discussion}

The dependence of the total energy on the unit cell volume of the two rare earth nitrides are investigated in the magnetic state in the two possible phases (RS, $\mathrm{CsCl}$ ), using the LSDA approximation. We found that RS structure is the most stable and the favorable structure of these compounds by minimization of energy as shown in Figs.(1 and 2). In agreement with the fact, that RS structure is the most stable and favorable structure of the rare earth nitrides compounds. Table (1), displays the calculated lattice constant of both compounds in the two structures. The equilibrium cell volume of $\mathrm{PmN}$ and $\mathrm{CeN}$ in the $\mathrm{RS}$ structure are 121.287 $\AA^{3}$ and $123.506 \AA^{3}$ respectively. In the present study, the magnetic phase of $\mathrm{PmN}$ in $\mathrm{RS}$ and $\mathrm{CsCl}$ structure is ferromagnetic. The calculated magnetic moments of $\mathrm{PmN}$ in $\mathrm{RS}$ and $\mathrm{CsCl}$ are $4 \mu_{\mathrm{B}}$ and $3.98 \mu_{\mathrm{B}}$ respectively. Only in the RS structure of PmN, we have found an energy gap of $0.56 \mathrm{eV}$ in the spin-down channel. The integer value of magnetic moment and the presence of the gap indicate that $\mathrm{PmN}$ is a half-metal compound in the $\mathrm{RS}$ phase. In contrast, the $\mathrm{CsCl}$ structure has zero energy gap, in both spin channels, which confirms the metallic behavior of this structure. Pandit et al [13] have checked out the presence of halfmetallicity in $\mathrm{CsCl}$ structure of $\mathrm{PmN}$, and found a small crossover of the d-band in the spin down channel at one point $(\mathrm{G})$. (However PmN is a semiconducting in all directions except this point in the spin-down channel). Olsen et al [23] have found the experimental lattice constant of $\mathrm{CeN}$ to be $5.021 \AA$ using the energy-dispersive $\mathrm{X}$-ray diffraction and synchrotron radiation using a diamond anvil cell at a maximum pressure of $77 \mathrm{GPa}$. They also used the full- potential linear muffin-tin orbital method within the GGA approximation to calculate the lattice constant and found it to be $5.024 \AA$. Our calculated lattice constant agrees well with both 
experimental and theoretical results of Olsen et al [23]. There are many authors who have calculated the lattice constant of $\mathrm{CeN}$ experimently e.g. Landelli et al [24] and Schlegel et al [25] who reported lattice constants of 5.021 $\AA$ and 5.019 respectively.

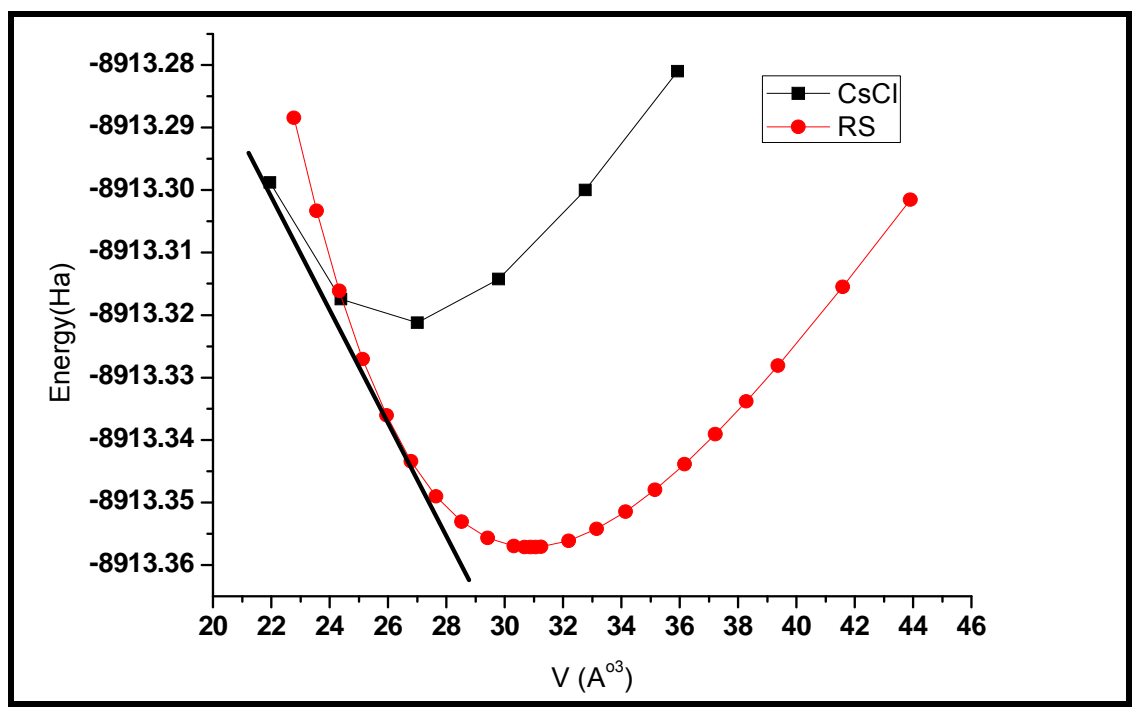

Fig.(1): The total energy dependence on the unit cell volume for magnetic CeN.

We have calculated the density of states and band structure of both PmN and $\mathrm{CeN}$ compounds in the $\mathrm{RS}$ and $\mathrm{CsCl}$ structures as shown in Figs.(3-5). We predicted from these Figures that:

1- The presence of an energy gap in the spin-down channel of PmN in the RS structure (Figs.3) confirms its half-metallic property.

2- $\mathrm{PmN}$ in $\mathrm{CsCl}$ structure and $\mathrm{CeN}$ in both $\mathrm{RS}$ and $\mathrm{CsCl}$ structures show a metallic behavior.

Table (1): The calculated lattice constant, magnetic moment, energy gap, and electronic state using LSDA approximation.

\begin{tabular}{|c|c|c|c|c|}
\hline \multirow{2}{*}{ Compound } & \multicolumn{2}{|c|}{$\mathrm{CeN}$} & \multicolumn{2}{c|}{$\mathrm{PmN}$} \\
\cline { 2 - 5 } & $\mathrm{RS}$ & $\mathrm{CsCl}$ & $\mathrm{RS}$ & $\mathrm{CsCl}$ \\
\hline \multirow{3}{*}{$\mathrm{a}(\AA)$} & 4.98 & & 4.95 & 2.95 \\
& $5.021^{[23]}$ & 3 & $4.984^{[12]}$ & $5.08^{[25]}$ \\
& $4.9^{[23]}$ & & $4.857^{[12]}$ \\
\hline $\mathrm{m}\left(\mu_{\mathrm{B}}\right)$ & 0.00 & \multirow{2}{*}{0.00} & $3.999^{[12]}$ & 3.98 \\
& $0.00^{[23]}$ & & 0.56 & 0.00 \\
\hline Energy Gap $(\mathrm{eV})$ & 0.00 & 0.00 & $\mathrm{H} . \mathrm{M}$ & $\mathrm{M}$ \\
\hline Electronic state & $\mathrm{M}$ & $\mathrm{M}$ & & \\
\hline
\end{tabular}




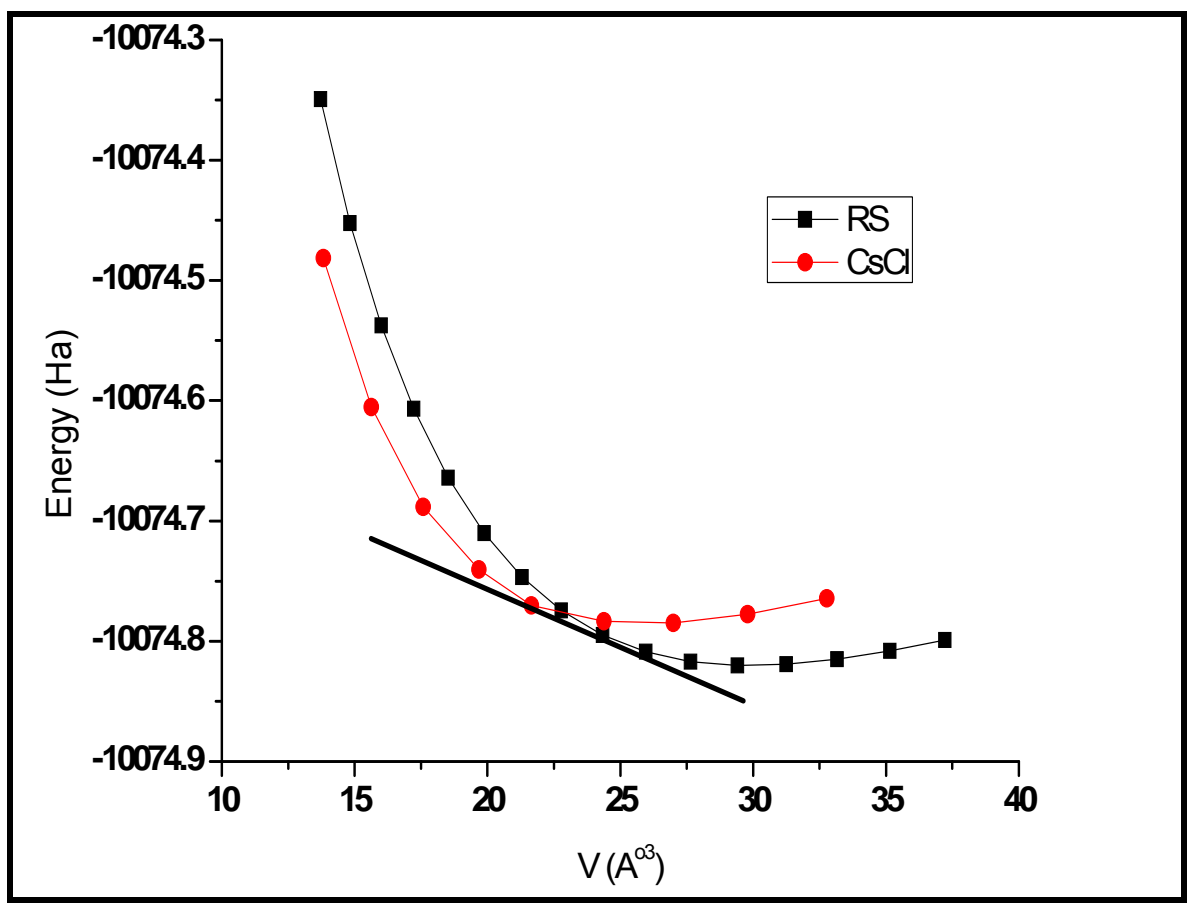

Fig.(2): The total energy dependence on the unit cell volume for magnetic PmN.

The bulk modulus and its pressure derivative are displayed in Table.(2). The bulk modulus of $\mathrm{CeN}$ compound is higher than the bulk modulus of $\mathrm{PmN}$ compound. The bulk modulus of $\mathrm{PmN}$ in $\mathrm{RS}$ and $\mathrm{CsCl}$ structures are 154.6 GPa and $113.96 \mathrm{GPa}$ respectively in good agreement with results of Pandit at al [12].

Table (2): The bulk modulus and its pressure derivative and phase transition pressure from $\mathrm{RS}$ to $\mathrm{CsCl}$ structure.

\begin{tabular}{|c|c|c|c|c|c|}
\hline Structure & \multicolumn{2}{|c|}{$\mathrm{RS}$} & \multicolumn{2}{c|}{$\mathrm{CsCl}$} & \multirow{2}{*}{$\begin{array}{c}\mathrm{Pt}(\mathrm{RS} \rightarrow \mathrm{CsCl}) \\
(\mathrm{GPa})\end{array}$} \\
\cline { 1 - 4 } Alloy & $\mathrm{B}_{\mathrm{o}}(\mathrm{GPa})$ & $\mathrm{B}_{1}$ & $\mathrm{~B}_{\mathrm{o}}(\mathrm{GPa})$ & $\mathrm{B}_{1}$ & $\begin{array}{c}40.7 \\
\mathrm{CeN}\end{array}$ \\
\hline \multirow{2}{*}{$\begin{array}{c}170.02 \\
210^{[23]}\end{array}$} & 4.39 & 180.33 & 1.087 & $62^{[11]}$ \\
\hline $\mathrm{PmN}$ & $\begin{array}{c}154.6 \\
150,165.6^{[12]}\end{array}$ & 5.93 & $\begin{array}{c}113.96 \\
90.83^{[12]}\end{array}$ & 4.98 & $\begin{array}{c}42 \\
\end{array}$ \\
\hline
\end{tabular}




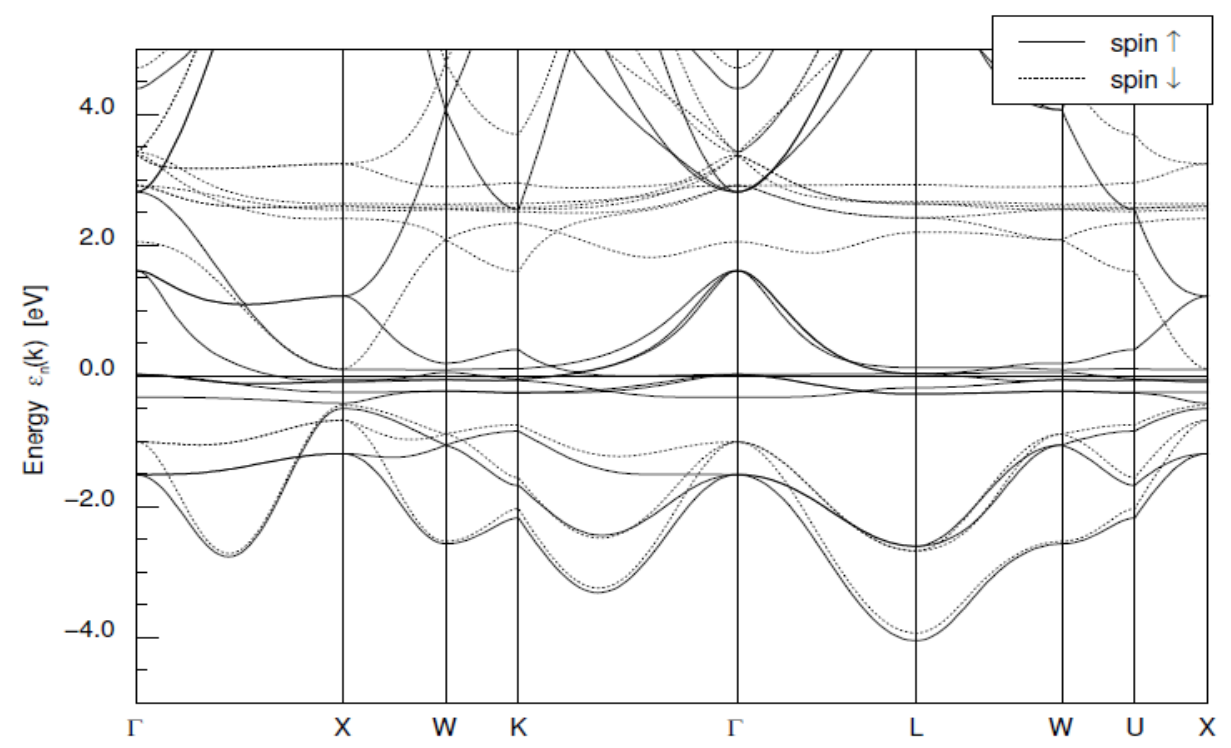

Fig.(3a): The band structure of $\mathrm{PmN}$ in the RS structure

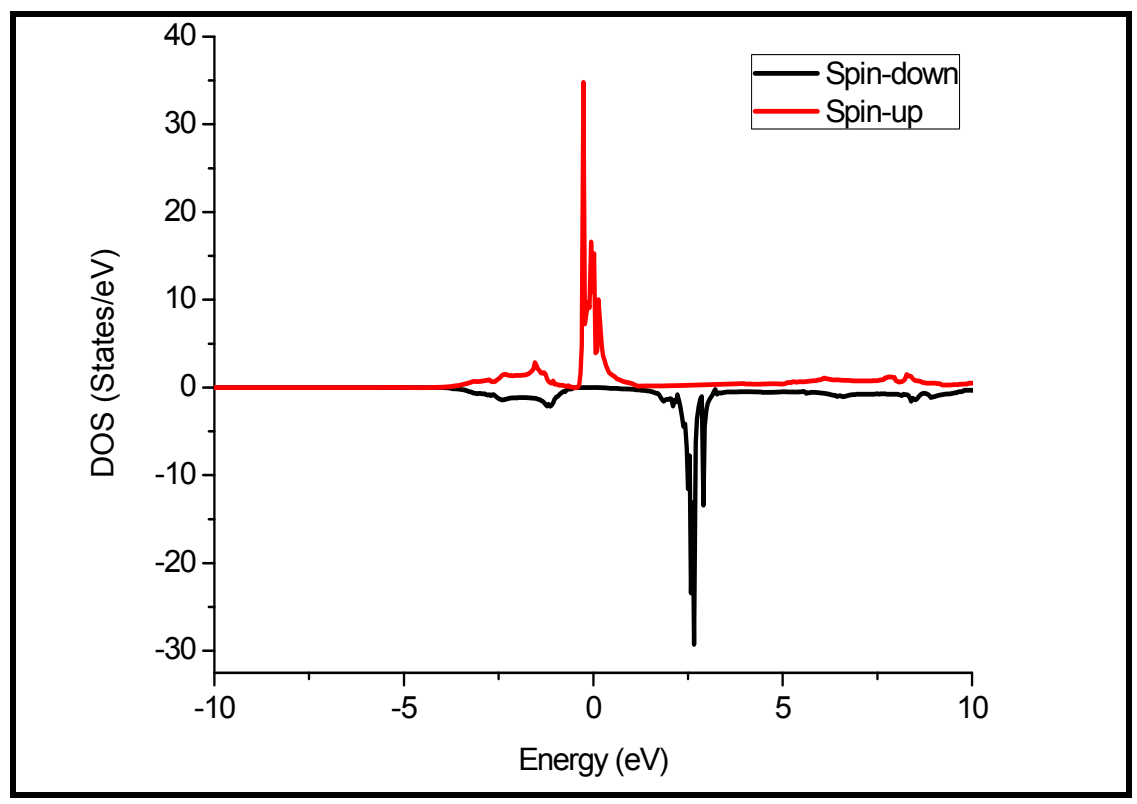

Fig.(3b): The DOS of PmN in the RS structure

We have investigated the structural phase transitions of $\mathrm{PmN}$ and $\mathrm{CeN}$ under hydrostatic pressure. A phase transition from $\mathrm{RS}$ into $\mathrm{CsCl}$ structure was predicted in both compounds at transition pressure of $42 \mathrm{GPa}$ and $40.7 \mathrm{GPa}$ respectively. This phase transition is predicted using the unit cell volume vs. pressure relationship as shown in Figs. (6 and 7) for $\mathrm{CeN}$ and $\mathrm{PmN}$ respectively. 


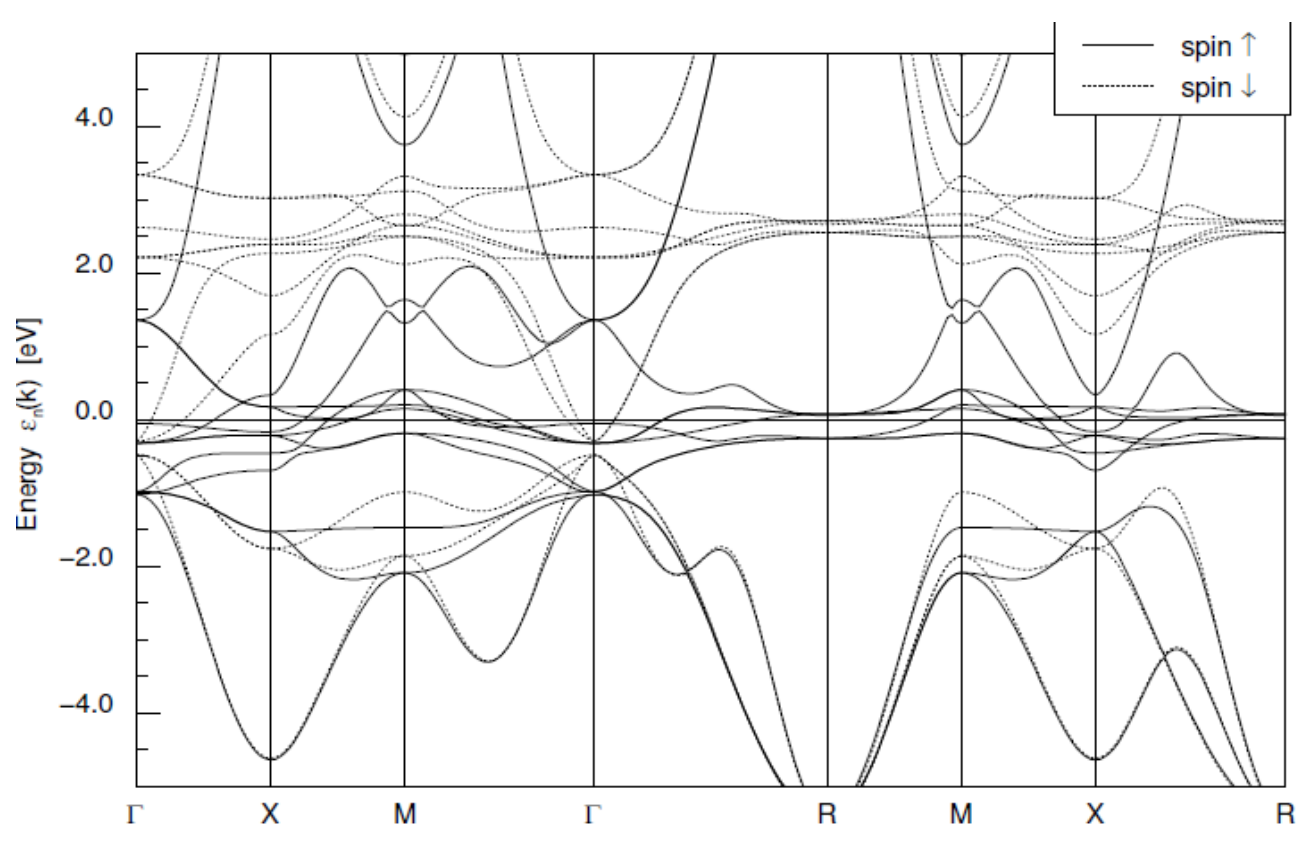

Fig.(4a): The band structure of $\mathrm{PmN}$ in the $\mathrm{CsCl}$ structure.

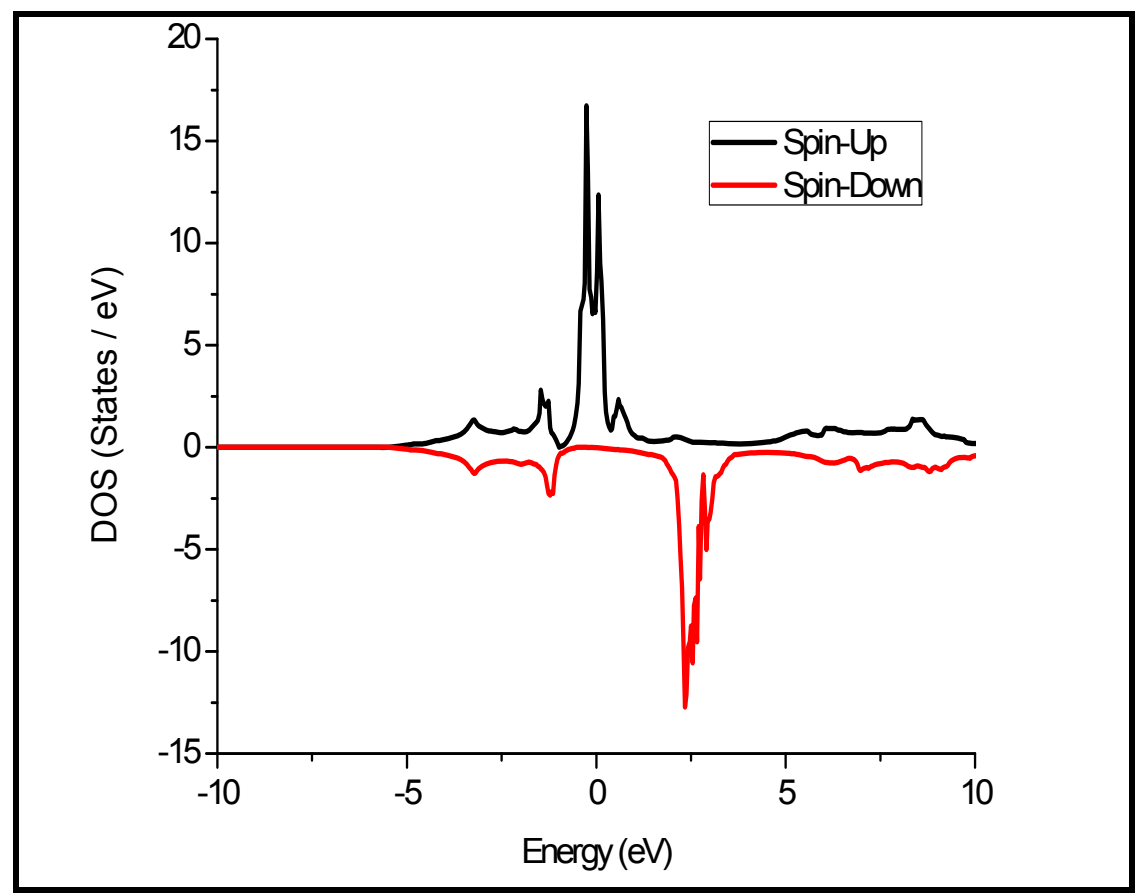

Fig.(4b): The DOS of $\mathrm{PmN}$ in the $\mathrm{CsCl}$ 


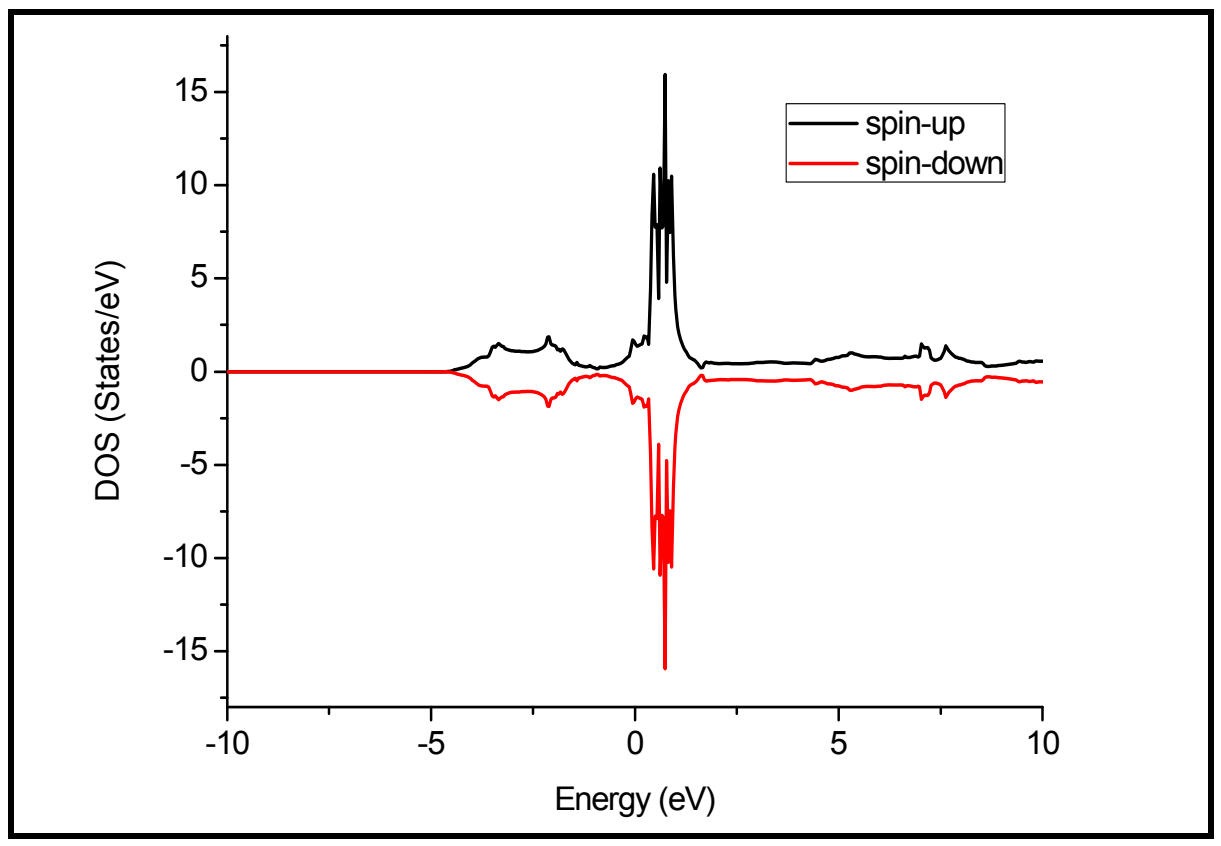

Fig.(5a): The DOS of CeN in RS

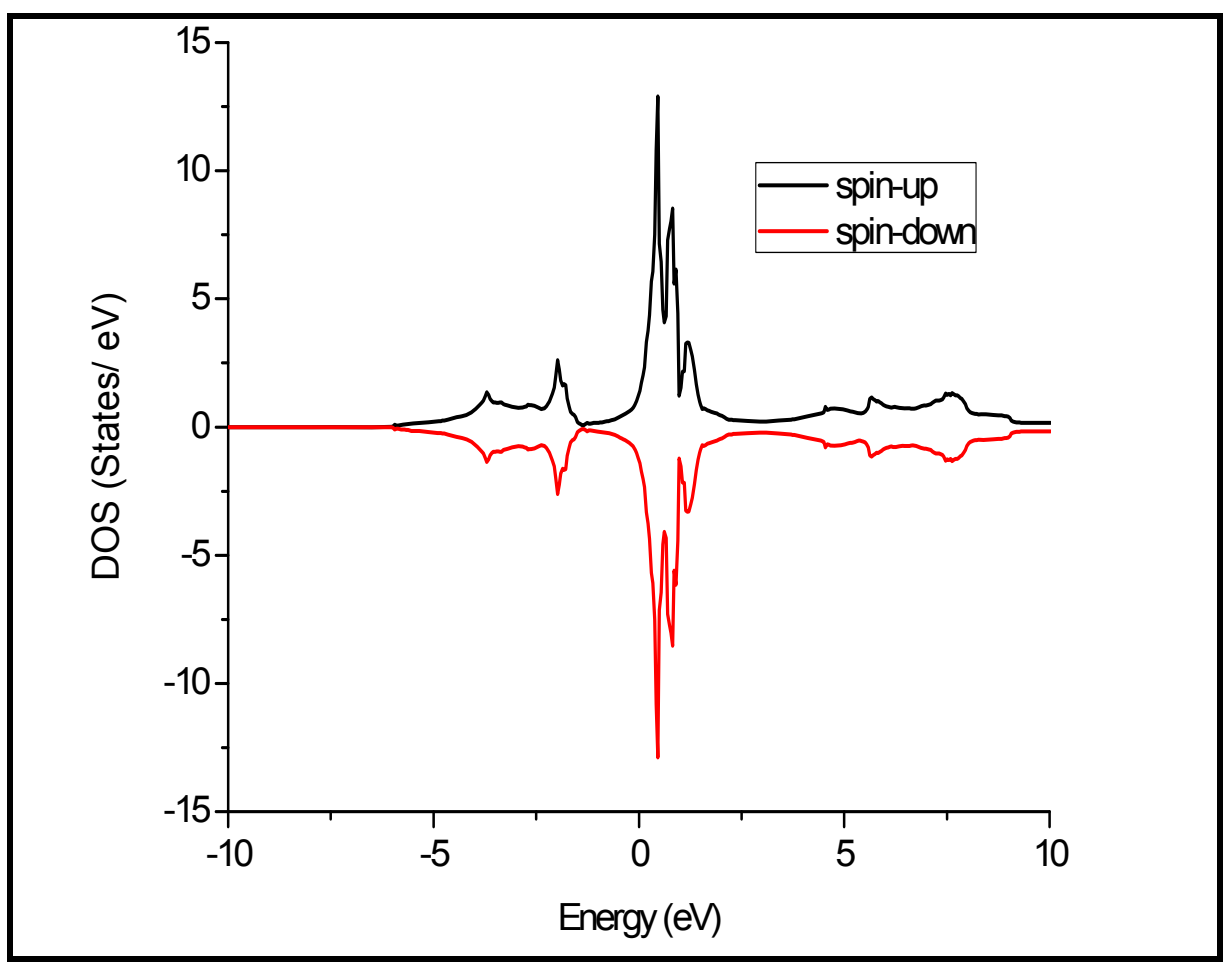

Fig.(5b): The DOS of $\mathrm{CeN}$ in $\mathrm{CsCl}$ 


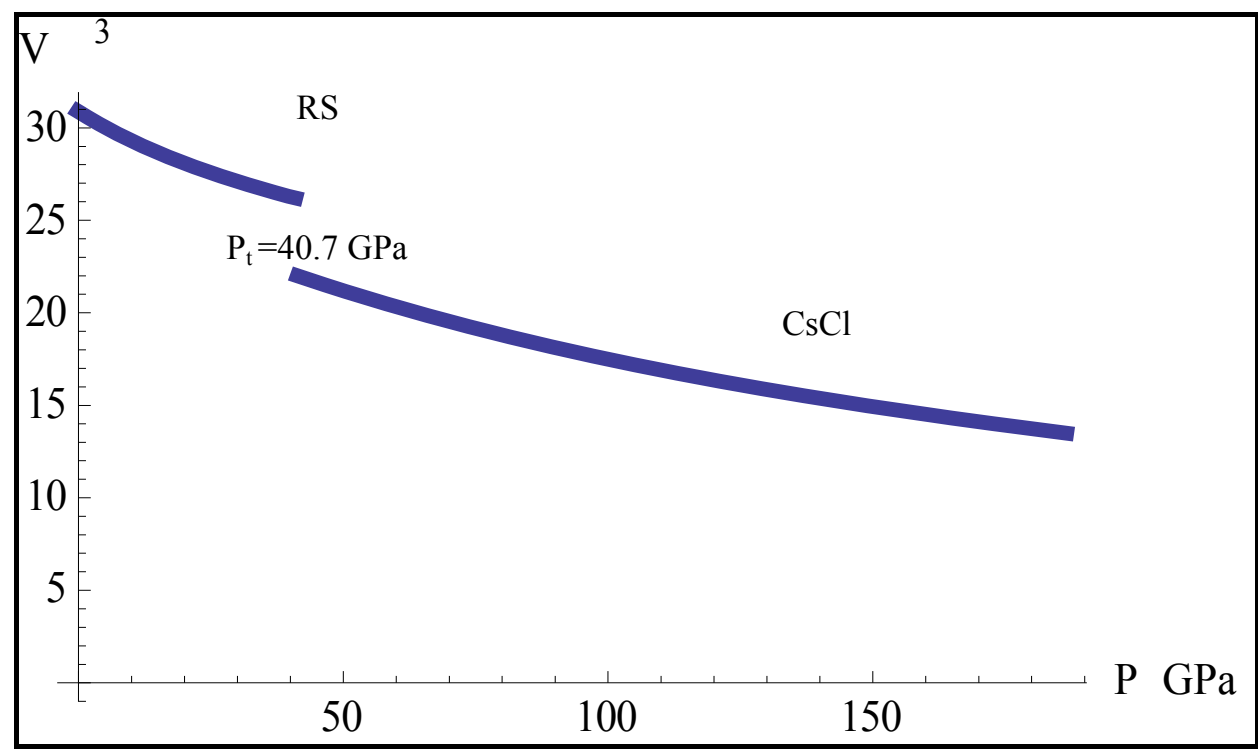

Fig.(6): The pressure vs. unit cell volume relationship showing the phase transitions from $\mathrm{RS}$ to $\mathrm{CsCl}$ structure in $\mathrm{CeN}$ compound.

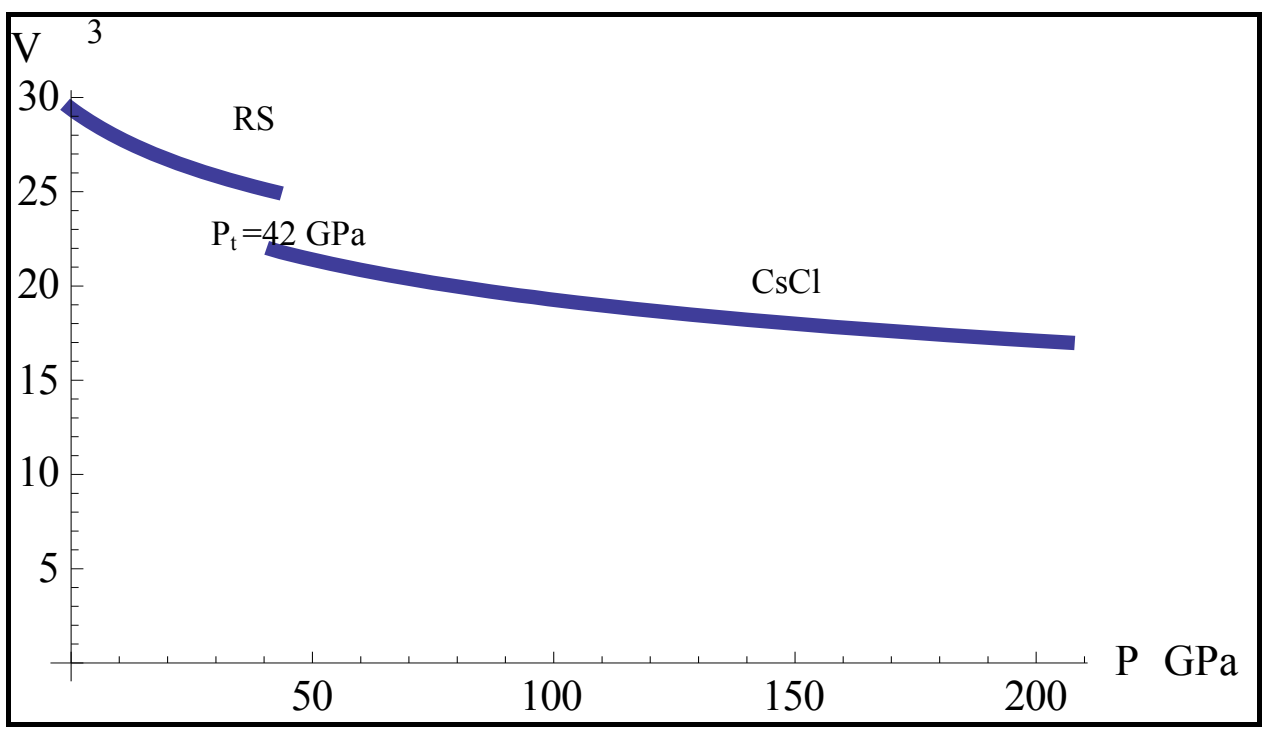

Fig.(7): The pressure vs. unit cell volume relationship showing the phase transitions from $\mathrm{RS}$ to $\mathrm{CsCl}$ structure in $\mathrm{PmN}$ compound.

\section{Conclusions}

Geometric optimization has indicated that the RS phase is energetically favorable in $\mathrm{PmN}$ and $\mathrm{CeN}$ compounds. We have studied $\mathrm{PmN}$ and $\mathrm{CeN}$ compounds in both $\mathrm{RS}$ and $\mathrm{CsCl}$ structures, and found that half metallicity appears only in the $\mathrm{RS}$ structure of $\mathrm{PmN}$ compound but the $\mathrm{CsCl}$ structure of $\mathrm{PmN}$ 
is metallic, and also $\mathrm{CeN}$ compound is metallic in both $\mathrm{RS}$ and $\mathrm{CsCl}$ structures. A phase transition was predicted in $\mathrm{CeN}$ and $\mathrm{PmN}$ from $\mathrm{RS}$ to $\mathrm{CsCl}$ at $\mathrm{P}=40.7 \mathrm{GPa}$ and $42 \mathrm{GPa}$ respectively. The bulk modulus and its pressure derivative for both compounds were calculated in the two structures.

\section{References}

1. S. Datta, B. Das, Appl. Phys. Lett., 56, 665 (1990).

2. K. A. Kilian, R. H.Victora, J. Appl. Phys., 87, 7064 (2000).

3. G. Vaitheeswaran, L. Petit, A. Svane, V. Kanchana and M. Rajagopalan, J. Phys. Condens. Matter, 16, 4429 (2004).

4. Shirotani, J. Hayashi, K. Yamanashi, K. Hirano, T. Adachi, N. Ishimatsu, O. Shimomura, and T. Kikegawa, Physica, B 334, 167 (2003).

5. G. Pagare, P. Soni, V. Srivastava, P. Sanyal, J. Phys. Chem. Solids, 70, 650 (2009).

6. Z. Szotek1, W.M. Temmerman, A. Svane, L. Petit, P. Strange, G.M.Stocks, D.K. odderitzsch, W. Hergert, H.Winter, J. Phys.: Condens. Matter, 16, s5587 (2004).

7. G. Pagare, V. Srivastava, P. Soni, S. P. Sanyal and M. Rajagopalan, J. Phys.: Conf. Series, 215, 012113 (2010).

8. R.G. Parr and W. Yang, Density Functional Theory of Atoms and Molecules (Oxford, Oxford, 1989).

9. R.M. Dreizler and E.K.U. Gross, Density Functional Theory: An Approach to the Quantum Many-Body Problem (Springer, Berlin, 1990).

10. A. Rukmangad, M. Aynyas, S.P. Sanyal, IJPAP, Vol. 47, 114 (2009).

11. A. Svane, Z. Szotek, W.M. Temmerman, H.W. Winter, Solid State Commun, 1202, 473 (1997).

12. P. Pandit, V. Srivastava, M. Rajagopalan, S.P. Sanyal, Physica B. 403, 4333 (2008).

13. V. Srivastava, M. Rajagopalan, S.P. Sanyal, J. MMM, 321, 607 (2009).

14. R. M. Shabara, S.Z. Yehia, S.H. Aly, Results in Physics, 1, 30 (2011).

15. S. Lundqvist, N. H. March, "Theory of the Inhomogenous Electron Gas", (Plenum, New York, 1983).

16. D. C. Langreth and J. P. Perdew, Phys. Rev. B 21, 5469 (1980).

17. http:/www.fplo.de

18. K. Koepernik and H. Eschrig, Phys. Rev., B 59, 1743 (1999).

19. M. A. Huleil, R.M. Shabara, S.H. Aly, M. Fadly, S.Z.Yehia, Advances in physics theories and Applications, ISSN 2222 (2013).

20. F. Birch, Phys. Rev., 71, 809 (1947).

21. F. D. Murnaghan, Am. J. Math., 59, 235 (1937).

22. F. D.Muraghan, Academy of Sciences, 30, 244 (1944).

23. G. L. Olcese, J. Phys. FiMet. phys., 9, 569 (1979).

24. A. Iandelli, E. Botti, Rendiconti Atti della Reale Accademia Nazionale dei Lincei 25, 129 (1937).

25. Schlegel, E. Kaldis, P. Wachter, C. Zürcher, Physics Letters A 66, 125 (1978). 